\title{
Oxidative stress and anti-oxidants in asymptomatic malaria-positive patients: a hospital-based cross-sectional Nigerian study
}

\author{
Ayodele S. Babalola ${ }^{1 *}$ (D) J. Jonathan ${ }^{2}$ and Boluwatife E. Michael ${ }^{2}$
}

\begin{abstract}
Background: Asymptomatic malaria is a threat to malaria eradication program. Host-related factors (e.g., immunity, oxidative stress, anti-oxidants activities) associated with asymptomatic malaria remain a gray area in research. This study seeks to determine the serum level of oxidative stress and anti-oxidants in 130 symptomatic and asymptomatic patients with different intensities of malaria parasite infection from a hospital in Ibadan, Nigeria.

Results: The prevalence of infection was $48.5 \%$ among the respondents. Most of the patients with parasitemia were asymptomatic (64.7\%). The mean titer value of malondialdehyde (MDA) was significantly higher $(p<0.05)$ among those with malaria infection (6.05 \pm 0.60$)$ compared with those who tested negative for malaria parasites (2.38 \pm 0.28$)$. Furthermore, the mean titer value of MDA was significantly higher $(p<0.05)$ among patients who showed symptoms of malaria (5.49 \pm 0.77$)$ compared with those without symptoms ( $2.93 \pm 0.47)$. A strong positive relationship existed between MDA $(r=0.717, p<0.05)$, glutathione peroxidase (GPx) $(r=0.695, p<0.05)$, and density of infection. On the other hand, a weak negative correlation existed between intensity and superoxide dismutase (SOD) $(r=-0.115, p>0.05)$ and glutathione (GSH) $(r=-0.278, p>0.05)$. The level of SOD and GSH also decreased significantly $(p<0.05)$ with an increase in MDA level.

Conclusions: This study showed that lipid peroxidation did not only increase in positive patients, it also rises in patients with clinical symptoms of malaria. Furthermore, a similar level of anti-oxidant responses was observed in both symptomatic and asymptomatic malaria patients. There is a need to inform health policies that encourage routine diagnosis and treatment of malaria in apparently healthy people if the malaria elimination goal is to be achieved in Africa.
\end{abstract}

Keywords: Asymptomatic, Malaria, Oxidative stress, Anti-oxidants capacity, Glutathione peroxidase, Superoxide dismutase

\footnotetext{
*Correspondence: ayodelebabalola2011@gmail.com;

babalola.ayodeles@pg.funaab.edu.ng

${ }^{1}$ Medical Parasitology Research Unit, Department of Pure and Applied

Zoology, Federal University of Agriculture, Abeokuta, Abeokuta, Nigeria

Full list of author information is available at the end of the article
}

\section{Springer Open}

(c) The Author(s). 2020 Open Access This article is licensed under a Creative Commons Attribution 4.0 International License, which permits use, sharing, adaptation, distribution and reproduction in any medium or format, as long as you give appropriate credit to the original author(s) and the source, provide a link to the Creative Commons licence, and indicate if changes were made. The images or other third party material in this article are included in the article's Creative Commons licence, unless indicated otherwise in a credit line to the material. If material is not included in the article's Creative Commons licence and your intended use is not permitted by statutory regulation or exceeds the permitted use, you will need to obtain permission directly from the copyright holder. To view a copy of this licence, visit http://creativecommons.org/licenses/by/4.0/. 


\section{Background}

Malaria has been in existence for so long and is responsible for worrisome health and economic impact in different parts of the world $[1,2]$. The devastating impact of malaria is more felt by the inhabitants of sub-Saharan Africa. Despite all efforts put in place by governments, donors, and researchers, malaria continues to persist, resulting in the deaths of children under five, pregnant women (who are the most vulnerable group), and even adults residing in sub-Saharan Africa [1, 2]. Malaria is caused by five species of parasites belonging to the genus Plasmodium, with P. falciparium being the most virulent and common in Africa including Nigeria.

The havoc caused by $P$. falciparium malaria in Africa cannot be overemphasized. The most frequent clinical manifestation of malaria infection is fever with other symptoms such as headache, nausea, vomiting, or diarrhea which appear 7-14 days following the bite of an infected female Anopheles mosquito [3]. When this happens, most people know that it is time to treat malaria either through self-medication or by medical personnel [4].

It is noteworthy that some studies have shown that some $P$. falciparium infections are without symptoms [5, 6]; however, the reason for this is not clear. While some school of thoughts believed it is more of the parasite factors (such as parasite density, rosetting and sequestration, toxin production, and genetic diversity including expression of virulence and variant antigen receptor), some believed that it could be more of host-related factors such as genes of the immune system, anti-oxidants, RBC polymorphisms or disorders, and circulating levels of immunoglobulins and cytokines [6]. The role of host factors in asymptomatic malaria largely remains a field ripe for inquiry [6].

Although "asymptomatic" malaria infections have no standard definition, it is generally referred to as malarial parasitemia of varying intensity, without fever or other acute symptoms, in individuals who have not received recent antimalarial treatment [1]. At any point in time, the vast majority of individuals with detectable malaria parasitemia can be categorized as asymptomatic based on this definition, regardless of the level of malaria density. This definition includes early detection of rising parasitemia that has yet to reach the pyrogenic threshold (i.e., the density of parasitized erythrocytes that is sufficient to trigger innate immune responses and fever) [1]. Asymptomatic malaria is prevalent in highly endemic areas of Africa and is a new challenge for malaria prevention and control strategies.

This research focused on oxidative stress and antioxidant levels which is also an important host-related factor when it comes to malaria parasite infection. Malaria parasitization is thought to increase oxidative stress, through the release of reactive oxygen species (ROS) in patients [7]. As a result, phagocytic cells such as polymorphonuclear leukocytes and macrophages usually engage in a respiratory burst as a host cell-mediated immune response, promoting free radical productions that react to yield ROS. The increase in lipid peroxidation (oxidative stress) level in malaria patients and a decrease in ascorbic acid and GSH (Anti-oxidants) have been observed to be accountable for the development of oxidative stress in malaria patients [8]. Hence, malaria parasite virulence seems to depend largely on the patients' antioxidant capacities, which in turn is determined by the concentrations of antioxidant micronutrients [10].

A study that examined the level of stress and antioxidants in both symptomatic and asymptomatic pregnant women [9] reported that no additional stress was observed among pregnant women with asymptomatic malaria. However, another study showed that physical and clinical conditions of asymptomatic parasitemic patients are not the same as those of symptomatic patients [10]. Despite these controversies, reports presenting the differences in oxidative stress and antioxidant levels among symptomatic and asymptomatic patients with malaria parasitemia are lacking. The dearth of information on this issue of great epidemiological importance formed the background against which this study was conducted, i.e., to determine the level of oxidative stress and antioxidants in symptomatic and asymptomatic patients with different intensities of malaria parasite infection in Ibadan, Nigeria.

\section{Methods}

\section{Description of the study location}

This study was carried out at Oranyan Primary Health Center, located at the Ibadan South East local government area of Oyo State, South-Western Nigeria. It is within longitude $7^{\circ} 7^{\prime} 34.5^{\prime \prime}$ North and $3^{\circ} 8^{\prime} 99.8^{\prime \prime}$ East.

\section{Criteria for selection and study population}

This study is cross-sectional, involving patients presenting themselves at the hospital for different purposes. Only patients with malaria symptoms such as fever (temperature $\geq 40.6{ }^{\circ} \mathrm{C}$ ), headache, chills (recurring every 2 to 4 days), loss of appetite, nausea, and vomiting (WHO [1]) and those without symptoms of malaria (apparently healthy patients) were approached for this study. Patients with more serious health issues such as diabetes and kidney problem were not selected for this study. Also, patients currently treating malaria or in the last 3 weeks as well as those with hematological disorders were excluded (all participants were newly diagnosed for malaria). Thus, a total of 130 patients were randomly selected (by ballot method, we selected those who picked group A and excluded those who pick group B) using a formula according to Yamane [11]. The 
formula used in calculating the sample size in this study (presented below) is a simplified formula for measuring a proportion within a specified margin of error sample according to Yamane [11]:

$$
n=\frac{N}{1+N e^{2}}
$$

where $n$ is the required sample size, $N$ is the total number of patients on the register for 3 months (193), and $e$ is the $5 \%$ margin of error.

Applying the above formula, the sample size was determined as presented below:

$$
n=\frac{193}{1+193\left(0.05^{2}\right)}
$$

Therefore, $n=130$.

Thus, a total of 130 patients were randomly chosen irrespective of age, gender, social class, marital status, and cultural or religious affiliations.

\section{Collection of blood samples and questionnaire administration}

Blood samples were collected from the participants between March and May 2019. Finger-pricked blood samples were collected for parasitemia level and PVC determination, while venous was collected into labeled plain bottles for evaluation of oxidative stress and antioxidant levels as described by Cheesbrough [12]. Relevant demographic characteristics, malaria history and treatment, health-seeking behaviors, and symptoms shown were recorded with the aid of a questionnaire.

\section{Laboratory procedures}

Thick and thin smears were prepared and stained with $10 \%$ Giemsa as described by Cheesbrough [12]. The blood films were examined microscopically using $\times 100$ objectives with oil immersion. Malaria diagnosis was based on the identification of asexual stages of Plasmodium spp. on the thick blood film, while the thin smears were used for species identification. Slides were declared negative after observing at least 100 high-power fields without detecting any parasites. Parasite density was calculated by estimating parasite numbers $/ \mu \mathrm{L}$ of blood from the thick film as described by Greenwood and Armstrong [13].

Packed cell volume (PCV) was determined using blood collected into heparinized capillary tubes and spun with a Hawksley micro-hematocrit centrifuge for $5 \mathrm{~min}$. Patients were classified as anemic and non-anemic using the WHO standard [14].

\section{Assay of oxidative stress marker}

Fresh blood samples were collected into labeled plain sample bottles and were centrifuged. The supernatant (serum) was then frozen for the oxidative stress enzyme analyses. The level of malondialdehyde (MDA) (a marker of free radical activity and lipid peroxidation) was determined using a spectrophotometer by reaction with thiobarbituric acid (TBA) in the plasma according to Okhawa et al. [15].

To determine glutathione peroxidase's (GPx) activity, $500 \mu \mathrm{L}$ of supernatant, phosphate buffer $(500 \mu \mathrm{L}), \mathrm{NaN}_{3}$ $(100 \mu \mathrm{L}), \mathrm{GSH}(200 \mu \mathrm{L}), \mathrm{H}_{2} \mathrm{O}_{2}(100 \mu \mathrm{L})$, and $600 \mu \mathrm{L}$ of distilled water were mixed. The whole reaction mixture was incubated at $37{ }^{\circ} \mathrm{C}$ for $3 \mathrm{~min}$ after which $0.5 \mathrm{ml}$ of TCA was added and thereafter centrifuged at $3000 \mathrm{rpm}$ for $5 \mathrm{~min}$. One milliliter of each of the supernatants, 2 $\mathrm{ml}$ of $\mathrm{K}_{2} \mathrm{HPO}_{4}$, and $1 \mathrm{ml}$ of DTNB were added, and the absorbance was read at $412 \mathrm{~nm}$ against a blank following the method of Rotruck et al. [16].

To determine the superoxide dismutase (SOD) activity, $100 \mu \mathrm{L}$ of buffer was added into the test and $150 \mu \mathrm{L}$ of buffer into the blank; $830 \mu \mathrm{L}$ of distilled water was pipette into both test tubes (test and blank), and $50 \mu \mathrm{L}$ of serum sample was added into the test. These were incubated at room temperature for $10 \mathrm{~min}$. Twenty microliters of pyrogallol was then added and immediately mixed by inversion, and the increase in absorbance at $340 \mathrm{~nm}$ for $3 \mathrm{~min}$ was recorded. $\Delta A 340 \mathrm{~nm} / \mathrm{min}$ was obtained following the method of Zou [17].

Lastly, glutathione (GSH) activity was determined following the method of Beutler et al. [18]. This method is therefore based upon the development of a relatively stable yellow color when 5,5-dithiobis-(2nitrobenzoic acid) (Ellman's reagent) is added to sulfhydryl compounds (present in the serum). The chromophoric product resulting from the reaction of Ellman's reagent with the reduced glutathione, 2nitro-5-thiobenzoic acid, possesses a molar absorption at $412 \mathrm{~nm}$. Reduced GSH concentration is proportional to the absorbance at $412 \mathrm{~nm}$.

\section{Statistical analysis}

Differences in the mean parasite densities, MDA, and anti-oxidant levels between symptomatic and asymptomatic malaria parasite-infected patients were tested using the Student $t$ test. Values were presented as mean \pm standard error of the mean. Relationships between parasite density and MDA as well as anti-oxidants were tested using correlation. Furthermore, variables that showed a strong and significant relationship with parasite density were further tested using a linear regression model. A probability value of $p<0.05$ was regarded as statistically significant, using SPSS version 20.0. 


\section{Results}

Prevalence of malaria parasite infection among the study participant

The prevalence of malaria parasite infection in the population was 63 of 130 (48.5\%). Furthermore, a significantly higher prevalence $(p=0.001)$ was recorded among the asymptomatic participants $33(64.7 \%)$ compared with those with malaria symptoms 30 (38.0\%). The mean parasite density was higher among symptomatic patients (4708.61 \pm 951.63$)$, compared to asymptomatic (3041.67 \pm 473.24). However, this does not reach a statistically significant level $(p=0.325)$.

Effect of malaria parasite infection on anemia among the study participants

The mean packed cell volume (PCV) was significantly higher $(p=0.001)$ among patients $(36.90 \pm 1.78)$ who tested positive for malaria parasites compared with those who tested negative $(27.80 \pm 1.45)$. However, there was no significant difference $(p=0.263)$ in PCV between patients who showed malaria symptoms and those who did not (Table 1).

The prevalence of anemia was significantly higher ( $p=$ 0.001 ) among those who tested positive for malaria parasite infection (63.5\%), compared to those that did not (26.9\%). Furthermore, the prevalence of anemia increased significantly $(p=0.013)$ with parasite density. Patients with low and high parasite densities recorded the least $(30 \%)$ and the highest $(81.8 \%)$ prevalence of anemia, respectively (Table 2 ).

\section{Relationship between oxidative stress markers and infection}

Malondialdehyde (MDA) values were significantly elevated $(p=0.000)$ in patients who tested positive for malaria parasite compared with those who tested negative. Furthermore, there was no significant difference in titer values of superoxide dismutase (SOD) and glutathione (GSH) among malaria-positive and malaria-negative patients in this study (Table 3), though SOD value was not significantly $(p=0.488)$ elevated in patients who tested positive for malaria parasite compared with those who tested negative. However, glutathione peroxidase values were significantly elevated $(p=0.002)$ in patients who tested positive for malaria parasite compared with those who tested negative (Table 3).

\section{Relationship between oxidative stress markers and malaria symptoms in infected patients}

Malondialdehyde (MDA) values were significantly elevated $(p=0.011)$ in patients who showed symptoms of malaria $\left(5.49 \pm 0.77 \mu / \mathrm{L} \times 10^{9}\right)$ compared with those who did not $\left(2.93 \pm 0.47 \mu / \mathrm{L} \times 10^{9}\right)$. Furthermore, there was no significant difference in the titer values of superoxide dismutase (SOD), glutathione peroxidase (GPx), and reduced glutathione (GSH) among those with symptomatic and asymptomatic malaria in this study (Table 4).

Relationship between parasite density, lipid peroxidation, and anti-oxidants in malaria parasite-infected patients $A$ strong, direct, and significant correlation existed between parasite density and MDA ( $r=0.717, p=0.001)$ as well as parasite density and GPx $(r=0.695, p=0.001)$ (Table 5). The regression equation showed that $\mathrm{MDA}=0.0007$ (density) $+3.3339\left(R^{2}=0.61\right)$ accounting for $61 \%$ of the variations observed in MDA with respect to parasite density. The regression equation showed that $\mathrm{GPx}=3 \mathrm{E}-05$ (intensity) $+0.1865\left(R^{2}=0.58\right)$ accounting for about $58 \%$ of the variations observed in GPx values (Fig. 1).

Furthermore, a weak, inverse relationship existed between both SOD $(r=-0.115)$ and GSH $(r=-0.278)$ and parasite density.

Furthermore, a strong, inverse, and significant relationship ( $p=0.002)$ existed between MDA and GSH $(r$ $=-0.641)$, while a very strong, direct, and significant relationship ( $p=0.0001)$ existed between MDA and GPx $(r=0.903)$. An inverse relationship existed between GSH and GPx $(r=-0.451)$ (Table 5).

\section{Discussion}

The close association between oxidative stress and diseases has become well known. Oxidative stress is defined as a "state in which oxidation exceeds the antioxidant

Table 1 Relationship between malaria parasite infection and mean PCV

\begin{tabular}{|c|c|c|c|}
\hline & Packed cell volume (mean $\% \pm \mathrm{SE}$ ) & $t$ values & $\overline{p \text { values }}$ \\
\hline \multicolumn{4}{|l|}{ Infection status } \\
\hline Negative & $36.90 \pm 1.78$ & 3.963 & $0.001^{*}$ \\
\hline Positive & $27.80 \pm 1.45$ & & \\
\hline \multicolumn{4}{|l|}{ Symptom status } \\
\hline Symptomatic & $30.60 \pm 2.67$ & -1.154 & 0.263 \\
\hline Asymptomatic & $34.10 \pm 1.43$ & & \\
\hline
\end{tabular}

*Significant difference $(p<0.05)$

Symptomatic patients were those with signs such as fever, headache, chills, loss of appetite, nausea, and vomiting according to WHO [1] 
Table 2 Effect of malaria parasite infection on the prevalence of anemia among the study participants

\begin{tabular}{|c|c|c|c|}
\hline \multirow[t]{2}{*}{ Variables } & \multirow{2}{*}{$\begin{array}{l}\text { No. of } \\
\text { examined, } \\
N(\%)\end{array}$} & \multicolumn{2}{|l|}{ Anemia status } \\
\hline & & Anemic, $N(\%)$ & Non-anemic, $N(\%)$ \\
\hline Malaria status & & & $p=0.001^{*}$ \\
\hline Positive & $63(48.5)$ & $40(63.5)$ & $23(26.5)$ \\
\hline Negative & $67(51.5)$ & $18(26.9)$ & $49(73.1)$ \\
\hline Total & $130(100.0)$ & $58(44.6)$ & $72(53.4)$ \\
\hline Malaria parasite density & & & $p=0.013^{*}$ \\
\hline Low & $10(15.9)$ & $3(30.0)$ & $7(70.0)$ \\
\hline Moderate & $42(66.7)$ & $23(54.8)$ & $19(44.2)$ \\
\hline High & $11(17.5)$ & $9(81.8)$ & $2(17.2)$ \\
\hline Total & $63(100.0)$ & $35(55.6)$ & $28(44.4)$ \\
\hline
\end{tabular}

*Significant difference $(p<0.05)$

systems in the body secondary to a loss of the balance between them" [19].

The increase in lipid peroxides has been recognized as an ultimate toxic effect of raised reactive oxygen species production by the immune system of the body, as well as a synchronized release of $\mathrm{O}_{2}{ }^{-}$during hemoglobin degradation by malarial parasites $[8,20]$. This study seeks to determine the level of lipid peroxidation and antioxidants in symptomatic and asymptomatic patients attending a primary health facility in Ibadan.

In this study, the prevalence of malaria parasite infection was $48.5 \%$. This means that malaria is still a problem in the study area. The prevalence observed in this study was higher than that of $[21,22]$ who reported a prevalence of $17 \%$ and $8.4 \%$, respectively, within the same province. In this study, most of the clients with parasitemia were asymptomatic. This is in line with the findings of $[5,22]$. This is attributable to the fact that Falciparium malaria can be asymptomatic in infected patients as part of adaptation to survival. Not feeling any symptoms may ultimately result in infected patients not seeking treatment against the deadly parasite.

Anemia was associated $(p<0.05)$ with malaria parasite infection of the peripheral blood. Malaria can cause anemia through the parasitic destruction of red blood cells (RBCs) and autoimmune reaction in which noninfected RBCs are also destroyed [23]. Furthermore, there was an obvious increase in the prevalence and severity of anemia with an increase in parasite density. It has been previously documented that in $P$. falciparum malaria, there is a direct correlation between an individual's asexual erythrocytic-stage parasite density at the time of presentation to a health care provider and the severity of clinical disease [24]. Patients with high parasite count have more severe and complicated course [24]. Oxidative stress is known to induce anemia through a chain of processes. The major target of oxidative stress is the red blood cell due to their primary function as $\mathrm{O}_{2}$-carrying cells [25]. Oxidative stressaffected erythrocytes produce molecular signals that lead to the activation of $\mathrm{Ca}^{2+}$-permeable cation channel, which facilitate $\mathrm{Ca}^{2+}$ entry into the cells. This eventually activates $\mathrm{Ca}^{2+}$-sensitive $\mathrm{K}^{+}$channels hereby leading to cell shrinkage and scrambling of the erythrocyte membrane. Next is the exposure of phosphatidylserine at the erythrocyte surface, typical signs of eryptosis (destruction of red blood cells) leading to severe anemia [25, 26].

Surprisingly, there was no significant difference in the packed cell volume among symptomatic and asymptomatic malaria-infected patients. This further buttresses

Table 3 Relationship between oxidative stress markers and infection

\begin{tabular}{|c|c|c|c|c|}
\hline Oxidative marker & Infection status & Mean values \pm SEM & $t$ values & $\overline{p \text { values }}$ \\
\hline \multirow[t]{2}{*}{ MDA $\left(\mu / L \times 10^{9}\right)$} & Negative & $2.38 \pm 0.28$ & -5.570 & $0.000^{*}$ \\
\hline & Positive & $6.05 \pm 0.60$ & & \\
\hline \multirow[t]{2}{*}{$\operatorname{SOD}(\mu / L)$} & Negative & $0.0097 \pm 0.00$ & 0.709 & 0.488 \\
\hline & Positive & $0.0096 \pm 0.00$ & & \\
\hline \multirow[t]{2}{*}{ GSH $(\mu / L)$} & Negative & $244.61 \pm 3.17$ & -1.546 & 0.156 \\
\hline & Positive & $249.53 \pm 0.24$ & & \\
\hline \multirow[t]{2}{*}{$\operatorname{GPx}(\mu / L)$} & Negative & $0.19 \pm 0.02$ & -3.611 & $0.002^{*}$ \\
\hline & Positive & $0.31 \pm 0.03$ & & \\
\hline
\end{tabular}

*Significant difference $(p<0.05)$ 
Table 4 Relationship between oxidative stress markers and malaria symptoms in infected patients

\begin{tabular}{|c|c|c|c|c|}
\hline Oxidative marker & Infection status & Mean \pm SEM & $t$ values & $p$ values \\
\hline \multirow[t]{2}{*}{$\operatorname{MDA}\left(\mu / L \times 10^{9}\right)$} & Symptomatic & $5.49 \pm 0.77$ & 2.827 & $0.011^{*}$ \\
\hline & Asymptomatic & $2.93 \pm 0.47$ & & \\
\hline \multirow[t]{2}{*}{$\operatorname{SOD}(\mu / L)$} & Symptomatic & $0.0096 \pm 0.00$ & -1.649 & 0.116 \\
\hline & Asymptomatic & $0.0098 \pm 0.00$ & & \\
\hline \multirow[t]{2}{*}{ GSH $(\mu / L)$} & Symptomatic & $249.17 \pm 0.14$ & 1.295 & 0.212 \\
\hline & Asymptomatic & $244.97 \pm 3.23$ & & \\
\hline \multirow[t]{2}{*}{ GPx $(\mu / L)$} & Symptomatic & $0.27 \pm 0.04$ & 0.843 & 0.410 \\
\hline & Asymptomatic & $0.23 \pm 0.02$ & & \\
\hline
\end{tabular}

*Significance difference $(p<0.05)$

Symptomatic patients were those with signs such as fever, headache, chills, loss of appetite, nausea, and vomiting according to WHO [1]

how dangerous asymptomatic malaria could be; while there are no visible signs of infection, a lot of damages would have been done by the "hidden" parasite. Therefore, it is important to understand the host-related factors that are responsible for this major setback.

As expected, the mean MDA and GPx titer values were higher among malaria-infected patients compared to noninfected patients. This is an implication that malaria patients had a significantly lowered total antioxidant capacity (TAC) than the uninfected control group. This might be due to the increased usage of the host's plasma antioxidants against the ROS generated by the malaria parasites to counteract oxidative stress. The oxidative stress marker estimated in this study was significantly higher in malaria patients than in the uninfected controls. This development will increase the oxidative stress index (OSI) which is the ratio of TOS to TAC and shows the exact degree of imbalance between oxidants and antioxidants in infected patients [31]. A compromised antioxidant defense mechanism, alongside increased oxidant levels and OSI values in malaria patients, might play an important role in the pathogenesis and severity of malaria [31]. Studies have shown that oxidative stress is common among malaria patients [25-27] as a result of the activation of the immune responses by the malaria parasite, thereby causing the release of reactive oxygen species (ROS) [28, 29]. Oxidative stress can occur when there is an imbalance of free radicals and antioxidants in the body [32]. Malarial infection is

Table 5 Pearson correlation coefficient between parasite density, lipid peroxidation, and anti-oxidants in malaria parasiteinfected patients

\begin{tabular}{llllll}
\hline & Density & MDA & SOD & GSH & GPx \\
\hline Density & 1 & & & & \\
MDA & $0.717^{*}$ & 1 & & & \\
SOD & -0.115 & -0.011 & 1 & & \\
GSH & -0.278 & $-0.641^{*}$ & 0.165 & 1 & \\
GPx & $0.695^{*}$ & $0.903^{*}$ & 0.262 & $-0.451^{*}$ & 1 \\
\hline
\end{tabular}

*Significance $(p<0.05)$
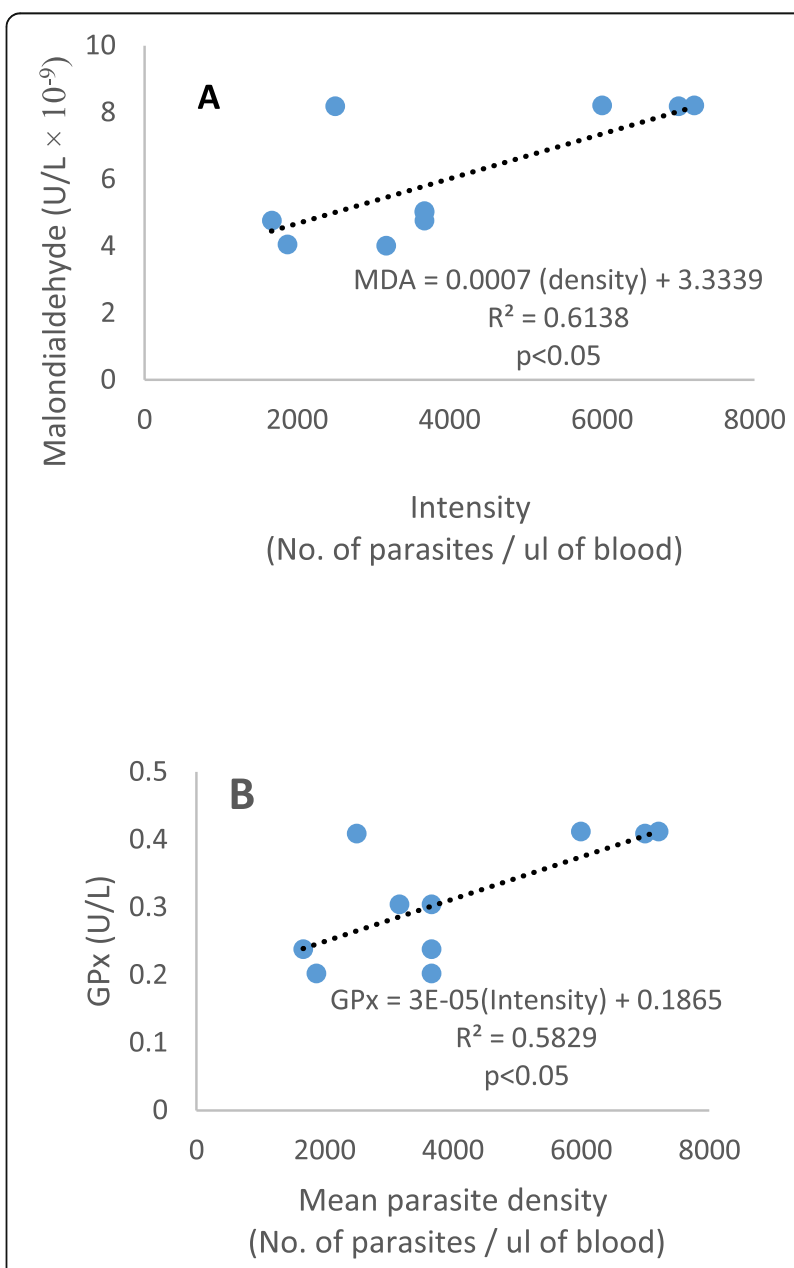

Fig. 1 Relationship between the mean parasite density and a MDA and $\mathbf{b}$ GPX 
associated with an increased production of reactive oxygen species by phagocytic cells. Changes due to toxic metabolites of the host and parasite may render the erythrocytes more vulnerable to damage [33].

Antioxidants are molecules which can safely interact with free radicals to terminate the chain reaction before vital molecules are damaged; if excess free radicals and oxidant give rise to a phenomenon known as oxidative stress, this is a harmful process that can negatively affect several cellular structures such as membrane lipids, proteins, lipoproteins, and deoxyribonucleic acid [33]. Antioxidants such as GSH, GPx, SOD, and catalase are known to play a major role in keeping the level of ROS in check during malaria infection [29]. Hence, this may explain the variations in the level of the anti-oxidants in infected and uninfected patients in this study.

In a similar Ethiopian study aimed to estimate the total oxidative stress and non-enzymatic antioxidant levels among 60 malaria patients and 40 healthy controls, levels of total oxidative stress (TOS), oxidative stress index (OSI), uric acid, albumin, and direct bilirubin levels were significantly increased in the serum of malaria patients compared to healthy control subjects [31]. On the other hand, the total antioxidant capacity and serum albumin levels were significantly reduced in malaria patients compared to the control groups. The study showed that the total oxidative status in malaria patients was significantly higher than that in control subjects [31].

The fact that a strong and direct relationship existed between parasite density and MDA as well as GPx buttresses the fact that malaria parasites are capable of inducing oxidative stress, and anti-oxidant levels (GPx) are equally being elevated to combat the effect of oxidative stress [30]. The inverse relationship between parasite density and SOD as well as GSH may be suggesting the using up of both antioxidants in fighting malaria-related oxidative stress [30].

MDA (a measure of oxidative stress) was elevated among symptomatic patients with malaria parasitemia compared with those who are not symptomatic. Oxidative stress is believed to be a key factor in the pathogenesis of malaria and contributes to the severity of malaria-related complications [34]. Malaria activates the immune system of the body causing the release of reactive oxygen species (ROS) like superoxide, hydrogen peroxide, hydroxyl radicals, lipid peroxides, and other related species. The parasite additionally fortifies certain cells and creates ROS via hemoglobin degradation [34]. A potential source of free radicals in malaria infection is the host's hemoglobin since the parasite utilizes hemoglobin for its own nutrition, liberating a lot of circulating heme. These heme groups can prompt intravascular oxidative stress, causing changes in erythrocytes and endothelial cells and encouraging the entry of the parasite in tissues, like the liver and cerebrum [35].

These findings may partly explain why a patient will show symptoms or not. The fact that there was no significant difference in all the anti-oxidant levels between symptomatic and asymptomatic patients is suggesting that there is an equal amount of anti-oxidant responses against the parasite in both symptomatic and asymptomatic infected host. This may suggest that the release of ROS (stress) as a result of infection may explain whether a patient will show symptoms of malaria or not. There is a need for further studies on factors that are responsible for malaria-related oxidative stress as well as asymptomatic malaria if the menace of malaria is to be curbed in Africa and other affected regions.

\section{Conclusion}

The prevalence of malaria parasite infection was $48.5 \%$, with most of the patients having parasitemia being asymptomatic. Anemia was associated $(p<0.05)$ with malaria parasite infection of the peripheral blood, and it increased with parasite density. Mean MDA and GPx titer values were higher among malaria-infected patients compared to non-infected. A strong and direct relationship existed between parasite density and MDA as well as GPx. While the antioxidant level has nothing to do with whether a host will show symptoms or not, the stress level of the host showed a significant relationship with malaria symptoms. Asymptomatic malaria is a threat to malaria eradication program; there is a need to inform health policies that encourage routine diagnosis and treatment of malaria in apparently healthy people if the malaria elimination goal is to be achieved in Africa

\section{Abbreviations \\ MDA: Malondialdehyde; GSH: Reduced glutathione; GPx: Glutathione peroxidase; SOD: Superoxide dismutase; $P$. falciparium: Plasmodium falciparium; PCV: Packed cell volume; ROS: Reactive oxygen species; OXI: Oxidative stress index; RNS: Reactive nitrogen species; TAC: Total antioxidant capacity; TOS: Total oxidative stress}

\section{Acknowledgements}

We will like to appreciate the staff of Oranyan Primary Health Center, Ibadan, for their help during the sampling collection. We also want to appreciate Mr. R.S.A for his assistance with the biochemical assays.

\section{Authors' contributions}

BAS conceived the idea for this study, did the statistical analysis, and wrote the manuscript. All authors carried out the literature search and research. BAS and JJ wrote the research protocol. All authors read and approved the final manuscript.

\section{Funding}

This research did not receive any specific grant from funding agencies in the public, commercial, or not-for-profit sectors

Availability of data and materials

The datasets generated during and/or analyzed during the current study are not publicly available due [general agreement among the authors] but are available from the corresponding author on reasonable request. 


\section{Ethics approval and consent to participate}

The Research and Ethics Committee of the hospital used provided ethical clearance (reference number 2019/UG/FUNAAB/15/1007/001) for the study. The health workers and the patients were fully briefed on the objectives of the study. Only participants who gave their consent (written consent) were enrolled in the study.

\section{Consent for publication}

Not applicable

\section{Competing interests}

The authors declare that they have no competing interests.

\section{Author details}

${ }^{1}$ Medical Parasitology Research Unit, Department of Pure and Applied Zoology, Federal University of Agriculture, Abeokuta, Abeokuta, Nigeria. ${ }^{2}$ Public Health Parasitology and Epidemiology Research Unit, Department of Pure and Applied Zoology, Federal University of Agriculture, Abeokuta, Abeokuta, Nigeria.

Received: 27 August 2020 Accepted: 22 October 2020

Published online: 04 November 2020

\section{References}

1. World Health Organization (2018) Malaria in children under five. WHO, Geneva Available online at: http://www.who.int/malaria/areas/high_risk_ groups/children/en/. Accessed 16 March 2020

2. WHO (2013) Water-related diseases. World Health Organization, Geneva Retrieved from: http://www.who.int/water_sanitation_ health/diseases/ malaria/en/ Accessed 16 March 2020

3. Howick VM, Russell AJC, Andrews T (2019) The Malaria Cell Atlas: single parasite transcriptomes across the complete Plasmodium life cycle. Science 365(6455):eaaw2619. https://doi.org/10.1126/science.aaw2619

4. Babalola AS, Idowu OA, Sam-Wobo FE (2015) Risk factors associated with the occurrence of placenta malaria among a population of parturients in Abeokuta. Malaria World J 6:8

5. Mlugu EM, Minzi O, Kamuhabwa AAR, Aklillu E (2020) Prevalence and correlates of asymptomatic malaria and anemia on first antenatal care visit among pregnant women in southeast, Tanzania. Int J Environ Res Public Health 17(9):3123. https://doi.org/10.3390/ijerph17093123

6. Shimizua S, Chotiratb C, Dokkulabb N, Hongchadb I, Khowsroyb K Kiattibutrb K, Maneechaib N, Manopwisedjaroen K, Petchvijitb P, Phumchueab K, Rachaphaewb N, Sripooroteb P, Suansomjitb C, Thongyodb W, Khamsiriwatcharac A, Lawpoolsrid S, Hanboonkunupakarna B, Sattabongkotb J, Nguitragoole W (2020) Malaria cross-sectional surveys identified asymptomatic infections of Plasmodium falciparum, Plasmodium vivax and Plasmodium knowlesi in Surat Thani, a southern province of Thailand. Int J Infect Dis 96(20):445-451

7. Eze MO, Hunting DJ, Ogan AU (1990) Reactive oxygen production against malaria - a potential cancer risk factor. Med Hypothesis 32:121-123

8. Atiku SM, Louise N, Kasozi DM (2019) Severe oxidative stress in sickle cell disease patients with uncomplicated Plasmodium falciparum malaria in Kampala, Uganda. BMC Infect Dis 19(1):600. https://doi.org/10.1186/s12879019-4221-y

9. Nwagha UI, Okeke TC, Nwagha TU (2011) Asymptomatic malaria parasitemia does not induce additional oxidative stress in pregnant women of South East Nigeria. Asian Pac. J. Trop. Med 4(3):229-233

10. Ogbodo SO, Okaka ANC, Nwagha UI (2014) Oxidative stress in symptomatic malaria parasitemic pregnant women from malaria endemic area of Nigeria. Am J Med Med Sci 4(5):168-174

11. Yamane T (1976) Statistics, an introductory analysis. 2nd ed. Harper and Row New York 1967

12. Cheesbrough M (2006) Medical laboratory manual for tropical countries, 3rd edn. Butterworths and Co (Publishers) Limited, Borough Green, pp 35-37

13. Greenwood BM, Armstrong JRM (1991) Comparison of two simple methods for determining malaria parasite density. Transactions of the Royal Society of Tropical Medicine and Hygiene 85:186-188

14. WHO (2011) Haemoglobin concentrations for the diagnosis and assessment of severity. Vitamin and Mineral Nutrition Information System. World Health Organization, Geneva (WHO/NMH/NHD/MNM/11.1). http://www.who.int/ vmnis/indicators/heamoglobin.pdf, accessed 15/02/2020
15. Okhawa H, Ohishi N, Yagi K (1979) Assay for lipid peroxides in animal tissues by thiobarbituric acid reaction. Anal Biochem 95:351-358

16. Rotruck JT, Pope AL, Ganther HE (1973) Selenium: biochemical role as a component of glutathione peroxidase. Science 179(4073):588-590

17. Zou GL (1986) Improvements in pyrogallol autoxidation method for the determination of SOD activity. J Agric Food Chem 4:71-73

18. Beutler E, Duron O, Kelly BM (1963) Improved method for the determination of blood glutathione. J Lab Clin Med 61:882-888

19. Yoshikawa T, Naito Y (2002) What is oxidative stress? J Jpn Med Assoc 124(11):1549-1553

20. Ifoue ST, Mofor CT, Gouado I (2009) Evaluation of oxidative stress and antioxidant status of pregnant women suffering from malaria in Cameroon. Ind J Clin Biochem 24(3):288-293

21. Anumudu Cl, Adepoju A, Adeniran M (2006) Malaria prevalence and treatment seeking behaviour of young Nigerian adults. Ann Afr Med 5(2): $82-88$

22. Falade CO, Olayemi O, Dada E (2008) Prevalence of malaria at booking among antenatal clients in a secondary health care facility in Ibadan, Nigeria. Afr JRep Health 12:141-152

23. Babalola AS, Idowu OA, Sam-Wobo SO, Fabusoro E (2017) Malaria infection at parturition in Abeokuta, Nigeria: current status and pregnancy outcome. Malaria World J 8:12

24. McElroy PD, Beier JC, Oster CN (1994) Predicting outcome in malaria: correlation between rate of exposure to infected mosquitoes and level of Plasmodium falciparum parasitemia. Am J Trop Med Hyg 51:523-532

25. Bissinger R, Bhuyan AA, Qadri SM, Lang F (2019) Oxidative stress, eryptosis and anemia: a pivotal mechanistic nexus in systemic diseases. FEBS J 286: 826-854. https://doi.org/10.1111/febs.14606

26. Lang KS, Duranton C, Poehlmann H, Myssina S, Bauer C, Lang F, Wieder T, Huber SM (2003) Cation channels trigger apoptotic death of erythrocytes. Cell Death Differ 10:249-256

27. Egwunyenga AO, Isamah G, Nmorsi OP (2004) Lipid peroxidation and ascorbic acid levels in Nigeria children with acute falciparum malaria. Afr $J$ Biotechnol 3:560-563

28. Kulkarni AG, Suryakar AN, Sardeshmukh AS (2003) Studies on biochemical changes with special reference to oxidant and antioxidants in malaria patients. Ind J Clin Biochem 18:136-149

29. Akanbi OM, Odaibo AB, Afolabi KA (2009) Anti-MSP-1(19) antibody (lgG) and reactive oxygen species (ROS) response against malaria infection in pregnancy in southwestern Nigeria. Asian Pac J Trop Med 2:9-15

30. Megnekou R, Djontu JC, Bigoga JD, Medou FM, Tenou S, Lissom A (2015) Impact of placental Plasmodium falciparum malaria on the profile of some oxidative stress biomarkers in women living in Yaoundé, Cameroon. PLoS One. 10(8):e0134633. https://doi.org/10.1371/journal.pone.0134633

31. Ebrahim A, Gnanasekaran N, Genet S (2019) Malaria patients correspond to increased parasitemia and severity of the disease. ROS 8(23):287-296 https://doi.org/10.20455/ros.2019.855

32. Pizzino G, Irrera N, Cucinotta M (2017) Oxidative stress: harms and benefits for human health. Oxid Med Cell Longev 2017:8416763. https://doi.org/10. 1155/2017/8416763

33. Lobo V, Patil A, Phatak A, Chandra N (2010) Free radicals, antioxidants and functional foods: impact on human health. Pharmacogn Rev. 4(8):118-126. https://doi.org/10.4103/0973-7847.70902

34. Sarr D, Cooper CA, Bracken TC, Martinez-Uribe O, Nagy T, Moore JM (2017) Oxidative stress: a potential therapeutic target in placental malaria. Immunohorizons. 1(4):29-41. https://doi.org/10.4049/immunohorizons. 1700002

35. Percário S, Moreira DR, Gomes BA, Ferreira ME, Gonçalves AC, Laurindo PS, Vilhena TC, Dolabela MF, Green MD (2012) Oxidative stress in malaria. Int Mol Sci 13(12):16346-16372. https://doi.org/10.3390/ijms131216346 PMID: 23208374; PMCID: PMC3546694

\section{Publisher's Note}

Springer Nature remains neutral with regard to jurisdictional claims in published maps and institutional affiliations. 